\title{
Nonlinear excitations match correlated motions unveiled by NMR in proteins: a new perspective on allosteric cross-talk
}

\author{
Francesco Piazza \\ Université d'Orléans, Centre de Biophysique Moléculaire, CNRS-UPR4301, Rue \\ C. Sadron, 45071, Orléans, France \\ E-mail: Francesco.Piazza@cnrs-orleans.fr
}

\begin{abstract}
In this paper we propose a novel theoretical framework for interpreting long-range dynamical correlations unveiled in proteins through NMR measurements. The theoretical rationale relies on the hypothesis that correlated motions in proteins may be reconstructed as large-scale, collective modes sustained by long-lived nonlinear vibrations known as discrete breathers (DB) localized at key, hot-spot sites. DBs are spatially localized modes, whose nonlinear nature hinders resonant coupling with the normal modes, thus conferring them long lifetimes as compared to normal modes. DBs have been predicted to exist in proteins, localized at few hot-spot residues typically within the stiffest portions of the structure. We compute DB modes analytically in the framework of the nonlinear network model, showing that the displacement patterns of many DBs localized at key sites match to a remarkable extent the experimentally uncovered correlation blueprint. The computed dispersion relations prove that it is physically possible for some of these DBs to be excited out of thermal fluctuations at room temperature.

Based on our calculations, we speculate that transient energy redistribution among the vibrational modes in a protein might favor the emergence of DB-like bursts of long-lived energy at hot-spot sites with lifetimes in the ns range, able to sustain critical, function-encoding correlated motions. More generally, our calculations provide a novel quantitative tool to predict fold-spanning dynamical pathways of correlated residues that may be central to allosteric cross-talk in proteins.
\end{abstract}

\section{Introduction}

Proteins are intrinsically dynamic machines. Their function, e.g. the ability to respond allosterically to a a local perturbation such as ligand binding or chemical modification [1], is intimately related to specific correlated vibrations, whose pattern is rooted in their native structure 22 6]. Although many experimental techniques lend considerable insight in protein dynamics $7-[0$, NMR spectroscopy is emerging as an increasingly powerful method for the characterization of correlated vibrational patterns involved in protein function and, notably, in allosteric intramolecular communication [11 17.

A large body of work in the paste decades has contributed to highlight the centrality of proteins' three-dimensional folds to their function. Protein scaffolds, for example, encode how energy flows across specific pathways encompassing certain 
hot-spot residues at key locations [18], and linking different functional regions, but also sometimes portions of structure not apparently associated with function [19, 20]. As it is well known, low-frequency, collective normal modes (NM) provide key foldencoded patterns for conformational changes associated with functional motions, very often in excellent agreement with observed conformational changes 21 24]. However, typically these modes are highly damped due to the substantial exposure to the solvent of large portions of the protein structure [25, 26], suggesting that other, more localized and therefore more robust higher-frequency vibrations might be implied in protein function.

The hypothesis that protein functional dynamics may imply the concerted action of large-scale motions sustained by specific higher-frequency localized vibrations, although not new [27, has been recently put forward 28] in the context of allostery, providing an intriguing rationale for the general idea that both slow and fast modes in proteins are connected to function 29 31. As already argued in the early 70s by C. McClare, for example, enzyme functioning may well imply non-thermal storage of energy in specific, fold-rooted localized vibrational modes, so as to lower the free-energy barriers of chemical reactions where it is needed 32 34. Indeed, many experiments have affirmed the crucial role of specific localized vibrations, such as hinge motions, in mediating between faster atomic fluctuations and slower functional rearrangements $35-37$.

Along the same lines, many experimental studies suggest that unusually longlived vibrational modes may be excited in proteins [38 41, reinforcing the idea that specific nonlinear effects may be central to their functional dynamics [27, 42, 45]. As a matter of fact, anharmonic effects are known since a long time to be highly relevant in many dynamical processes in proteins [38, 46, 49]. For example, recent experiments show that the activation of anharmonic modes is required for enzymatic activity in Lysozyme [50].

More recently, it has been shown that nonlinear excitations known in many systems as Discrete Breathers (DB) allow one to cleverly dissect hot-spot sites and intramolecular signaling pathways of connected residues critical to protein functioning [51; 52]. DBs are time-periodic, spatially localized vibrational modes that are found generically in many-body nonlinear systems [53] and possess many properties that make them interesting in the context of protein dynamics and allosteric communication. It has been shown that DBs promote and sustain long-range dynamical cross-talk in proteins, mediating energy transfer to distant locations [54]. DBs are able to self-stabilize by harvesting energy from the background, which has also been directly related to DB-mediated long-range communication across protein structures [52; 55]. In fact, at variance with topological excitations such as solitons, interactions between DBs or between a DB and a large vibrational energy fluctuation generally cause a substantial flow of energy from the less energetic to the more energetic mode. Remarkably, this sheer nonlinear phenomenon has been reported to put distant regions of protein structures in communication f following isolated energy kicks at specific locations that trigger the spontaneous emergence of a $\mathrm{DB}$ at a distant site [51. Moreover, by construction DB frequencies do not resonate with normal modes, which hampers DB-NM resonant transfer and makes DBs robust

$\ddagger$ Although it seems that the link between energy harvesting and transfer is indeed related to the emergence of short-lived nonlinear modes (or large energy fluctuations) at key passage sites [51] (making up the energy transduction pathway), the exact mechanisms underlying such kind of longrange, spontaneous energy-harvesting phenomena is still unclear. 
against perturbations. DBs are also protected from solvent-mediated instabilities, as their hot spots lie at locations typically far from the protein surface [55. For all these reasons, DBs appear as ideal candidates to realize specific long-lived, highly-correlated and fold-spanning motions featuring high resilience to perturbations. It should be stressed that, in view of their nonlinear character, DBs, despite being localized in space, are true collective motions, where all the particles in the system vibrate at one and the same frequency [53. This is the deep reason why DB-based analyses are able to unveil fold-rooted dynamical pathways of correlated residues over the entire scaffold of a protein [51.

NMR spectroscopy has proved an invaluable tool to dissect long-range correlations in proteins [56; 57. Recently, methods based on statistical analysis of NMR chemical shift changes [11; 12] and perturbation patterns [58] have been introduced, showing a great potential of mapping extended networks of coupled amino acids involved in intramolecular signaling pathways [59. In particular, a recent NMR study reported residual dipolar couplings measurements revealing a rather puzzling long-lived correlated motion spanning four $\beta$ strands separated by up to $1.5 \mathrm{~nm}$ in Ubiquitin [14], showing clear signatures on the msec time scale and raising intriguing questions as to the nature of the associated vibrational mode(s). The observed correlations resulted from large-scale concerted conformational rearrangements partly mediated by the hydrogen-bonding network. The structures of 640 conformers have been deposited in the PDB repository following this study (PDB identifier 2KOX), providing a wealth of precious structural information.

In this paper we show that the vibrational pattern of several distinct discrete breather modes found in Ubiquitin at specific key residues match to an amazing extent the correlated pattern uncovered experimentally. Furthermore, we show that the energy required for exciting such modes could in principle be available through thermal energy fluctuations occurring at key sites, typically residing in the stiffest locations of the protein fold. One the one hand, our results strongly suggest that the correlations observed experimentally in Ref. 14] could flag the spontaneous excitation of discrete breathers in Ubiquitin. More generally, our calculations suggest that DB-based methods may be central to unveil sub-structures comprising residues that mediate the transmission of allosteric signals in proteins.

The paper is organized as follows. First we introduce our coarse-grained model of protein dynamics and describe the essentials of our algorithm for computing approximate analytic DB solutions. We then illustrate, in the case of a randomly selected conformer, how DBs localized at specific sites match the experimental correlated pattern. Finally, we report the results of the analysis performed over the whole ensemble of NMR conformers, which confirms that the same results apply to DBs computed in all experimentally resolved structures.

\section{Analytic DB modes in the nonlinear network model}

We introduced the Nonlinear Network Model (NNM) with the aim of exploring the subtle effects arising in many-body systems from the interplay of anharmonicity and the lack of translational order (i.e. the peculiar $3 D$ protein folds) [55, for which little is known [60 62 as opposed to nonlinear systems with lattice-like translational invariance [63. In the NNM a given protein is coarse-grained to the level of aminoacids and modeled as a nonlinear network of $N$ identical point-like particles of mass $M \approx 110$ a.m.u. (i.e. the average amino-acid mass) placed at the corresponding $C_{\alpha}$ 
sites. The NNM potential energy reads

$$
V=\sum_{i>j} c_{i j}\left[\frac{k_{2}}{2}\left(r_{i j}-R_{i j}\right)^{2}+\frac{k_{4}}{4}\left(r_{i j}-R_{i j}\right)^{4}\right]
$$

where $r_{i j}=\left|\boldsymbol{r}_{i}-\boldsymbol{r}_{j}\right|$ is the distance between particles $i$ and $j$ and $R_{i j}=\left|\boldsymbol{R}_{i}-\boldsymbol{R}_{j}\right|$ is the $i-j$ separation in the equilibrium structure (in this case one of the NMR conformers). Here $\boldsymbol{r}_{i}$ and $\boldsymbol{R}_{i}$ denote the instantaneous and equilibrium position vectors of particle $i$, respectively. The connectivity matrix is simply $c_{i j}=\left\{1\right.$ if $R_{i j} \leq R_{c} \mid 0$ otherwise $\}$, where $R_{c}$ is a cutoff that identifies the interacting pairs. The aim being to explore the connection between nonlinearity and the peculiar structural features of the native folds, only $\mathrm{C}_{\alpha}$ atoms are taken into account and $k_{2}$ is assumed to be the same for all interacting pairs. Following our previous studies [55, we take $R_{c}=10 \AA$, and fix $k_{2}$ so that the low-frequency part of the linear spectrum match the corresponding measured frequencies. This gives $k_{2}=5 \mathrm{kcal} / \mathrm{mol} / \AA^{2}$. Furthermore, we fix $k_{4}=5$ $\mathrm{kcal} / \mathrm{mol} / \AA^{4}$, which corresponds to a rather weak nonlinearity $\S$. From a physical point of view, the constants $k_{2}$ and $k_{4}$ can be rationalized in terms of an average inter-residue potential of mean force (PMF). For a given pair of amino acids, a PMF for a given reaction coordinate (in our case the $\mathrm{C}_{\alpha}-\mathrm{C}_{\alpha}$ distance) can be computed, e.g. via Boltzmann inversion from all-atom molecular dynamics simulations. The idea here is that $k_{2}$ and $k_{4}$ measure the coefficients of the second-order and fourthorder terms in a Taylor expansion of such PMF. It is interesting to note that reaction coordinates associated with side-chain side-chain relative positions (e.g. the distance between centers of mass) are likely to yield strongly anharmonic (flat) PMFs. This is a consequence of the strongly nonlinear (multimodal) character of side-chain motions as revealed by the dynamics of $\chi_{1}$ dihedral angles [27].

In ref. 64] we have introduced a theoretical protocol for calculating analytically DB modes of given amplitude $A$ at a given site in the framework of the NNM. In principle, like all time-periodic functions, a DB solution can be decomposed as a Fourier series comprising harmonics of a fundamental frequency. However, The idea is to start from an ansatz for the DB consisting of the simplest time-periodic function modulated by a time-varying amplitude

$$
\boldsymbol{r}_{m}(t)=\boldsymbol{R}_{m}+A \boldsymbol{\xi}_{m}(t) \cos \omega t
$$

where we assume that the spatially localized envelope function $\boldsymbol{\xi}_{m}(t)$ varies slowly on the timescale defined by the inverse DB frequency $\omega^{-1}$. This fact can be simply pictured as an alternative statement of DB resilience to perturbations 53 . Moreover, we also assume that $\max _{m} \boldsymbol{\xi}_{m}(t) \simeq \mathcal{O}(1)$, so that the parameter $A$ sets the physical scale for the oscillation amplitude. Under these hypotheses, we can substitute ansatz (2) in the equations of motion and expand the forces in power series of $\epsilon_{i j}=A / R_{i j}$

$$
M \ddot{\boldsymbol{r}}_{m}=-\sum_{p=1}^{3} \frac{1}{p !}\left[\frac{\partial^{p}}{\partial A^{p}} \boldsymbol{\nabla}_{m} V\right]_{A=0} A^{p}+\mathcal{O}\left(\epsilon^{4}\right)
$$

We then multiply Eqs. (3) by cos $\omega t$ and average over one DB period. Under the slowly-varying envelope approximation, we can thus eliminate the time dependence, i.e.

$$
\left\langle\boldsymbol{\xi}_{m}(t) \cos ^{p} \omega t\right\rangle_{\omega} \approx \overline{\boldsymbol{\xi}}_{m}\left\langle\cos ^{p} \omega t\right\rangle_{\omega}
$$

$\S$ For $\left|r_{i j}-R_{i j}\right| \approx 0.5 \AA$ the nonlinear-to-linear energy ratio is $k_{4}\left|r_{i j}-R_{i j}\right|^{2} / 2 k_{2} \simeq \mathcal{O}\left(10^{-1}\right)$ 
where $\langle f\rangle_{\omega}=\omega / 2 \pi \int_{0}^{2 \pi / \omega} f(t) d t$ denotes the time average. Neglecting by the same token the second time derivatives of the functions $\boldsymbol{\xi}_{m}(t)$, we finally map the original set of differential equations onto a nonlinear system of $3 N$ algebraic equations, whose unknowns are the time-averaged envelope patterns $\overline{\boldsymbol{\xi}}_{m}$ (normalized to the displacement of the central particle) and the breather frequency. A DB mode with given amplitude $A$ is then found by numerically solving the algebraic system corresponding to an appropriate initial guess. We have found that the local direction of maximum stiffness provides an excellent initial guess, ensuring fast convergence and allowing one to investigate the parameter regions where a DB mode localized at a given site can be found 64].

\section{Nonlinear correlated motions in Ubiquitin}

A general structural analysis proves useful as a start, as this will provide an instructive reading frame for the results illustrated in the rest of the paper. The PDB file labelled $2 \mathrm{KOX}$ contains 640 possible conformers of ubiquitin. Thus, in principle a NNM can be constructed starting from each of those structures and analyzed separately. We have shown that few DBs localized at specific key sites, usually within stiff locations, appear to be far more stable and robust than all other DBs III. Such DBs arise as nonlinear continuations of high-frequency normal modes, which are spatially localized too, and invariably feature gap-less excitation spectra at variance with DBs localized at generic sites 64]. This means that we can gain valuable insight by examining the patterns of hot-spot sites highlighted by high-frequency NMs within the structural network of the protein over the whole NMR ensemble.

For each conformer, we construct a network as indicated above and compute the normal modes, i.e. the eigenvectors of the Hessian matrix of the total potential energy $V$ (1). Fig. 1 reports the pattern of occurrence of the NM sites of the first two highfrequency NMs, i.e. the particles whose amplitude of vibration in the mode is largest. It is apparent that a few sites stand out over the whole ensemble, which identify rigid, hinge-like locations [55. These sites lie within the stiffest regions and are thus subject to small-amplitude fluctuations within the ensemble of conformers. Overall, hot-spot sites are identified around LYS 27, LEU 50 and LEU 69. Interestingly, according to NMR measurements reported in Ref. [14, the last two sites appear to participate to the correlated motion uncovered in the experiment. More precisely, the latter identify the following subset of residues (see also cartoon in Fig. 1)

$$
A=\left\{\begin{array}{ll}
\text { VAL } 5-\text { THR } 7, & \text { LYS } 11-\text { ILE 13, } \\
\text { LEU } 43-\text { PHE } 45, & \text { LYS } 48-\operatorname{LEU~} 50, \\
\text { LEU } 67-\operatorname{LEU~} 69 &
\end{array}\right\}
$$

Our analysis over the ensemble of conformers clearly singles out three different regions as possible localization hot-spots for nonlinear localized modes. Thus, the question naturally arises as to what is the correlated pattern associated with DBs localized at these special locations.

Figures 2 (a) and (b) show the theoretical displacement patterns of two DB modes centered at LEU 67 and LEU 69. These have been calculated within the NNM starting from the equilibrium structure corresponding to a randomly picked conformer within the NMR ensemble (equivalent pattern maps are obtained by selecting other

|| Different measures of local stiffness can be adopted (see Ref. 55 for a possible definition), but the substance of this observation does not change. 


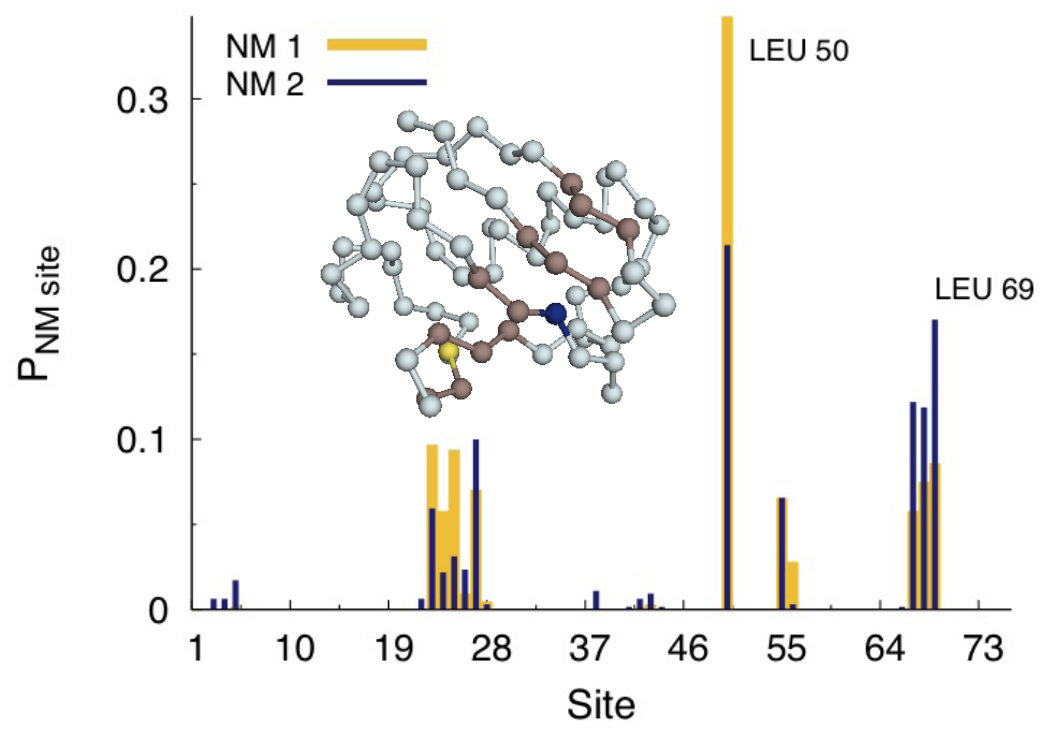

Figure 1. (Color online) Probability that a residue be the NM site (i.e. the particle with the largest amplitude) of the highest- or second-highest-frequency normal mode. The cartoon shows a $\mathrm{C}_{\alpha}$-coarse-grained structures of Ubiquitin. The region spanning the $\beta$ strands where the correlated motion is detected in the NMR experiments is highlighted in brown. Residues LEU 50 and LEU 69 are shown as yellow and dark blue spheres, respectively. The cutoff used to calculate the connectivity matrices is $R_{c}=10 \AA$.

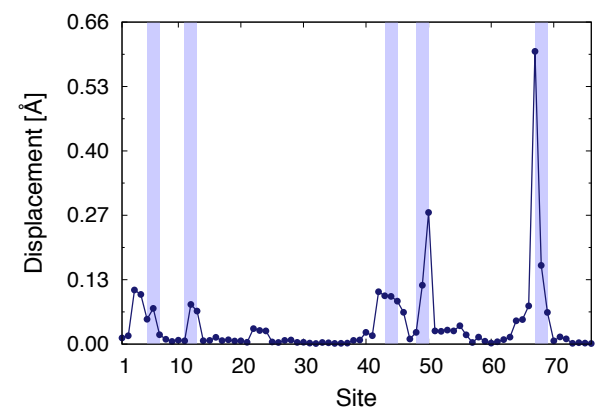

(a)

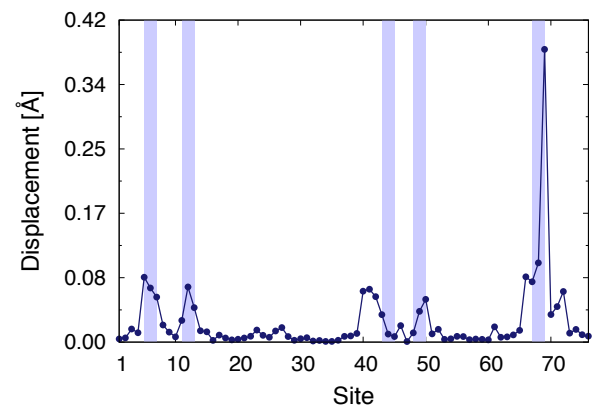

(b)

Figure 2. (Color online) Displacement patterns of DBs in the $400^{\text {th }}$ conformer of the NMR-resolved Ubiquitin structure. The residues whose correlated motion has been revealed by the NMR measurements from ref. [14] (see 5) lie within the light blue bands. (a) DB at site LEU $67, \omega_{B}=90.1 \mathrm{~cm}^{-1}, E_{B}=21.4 \mathrm{kcal} / \mathrm{mol}$. (b) DB at site LEU $69, \omega_{B}=86.2 \mathrm{~cm}^{-1}, E_{B}=7.7 \mathrm{kcal} / \mathrm{mol}$. Here $E_{B}$ and $\omega_{B}$ denote the potential energy associated with the displacement field of the $\mathrm{DB}$ and its frequency, respectively. 


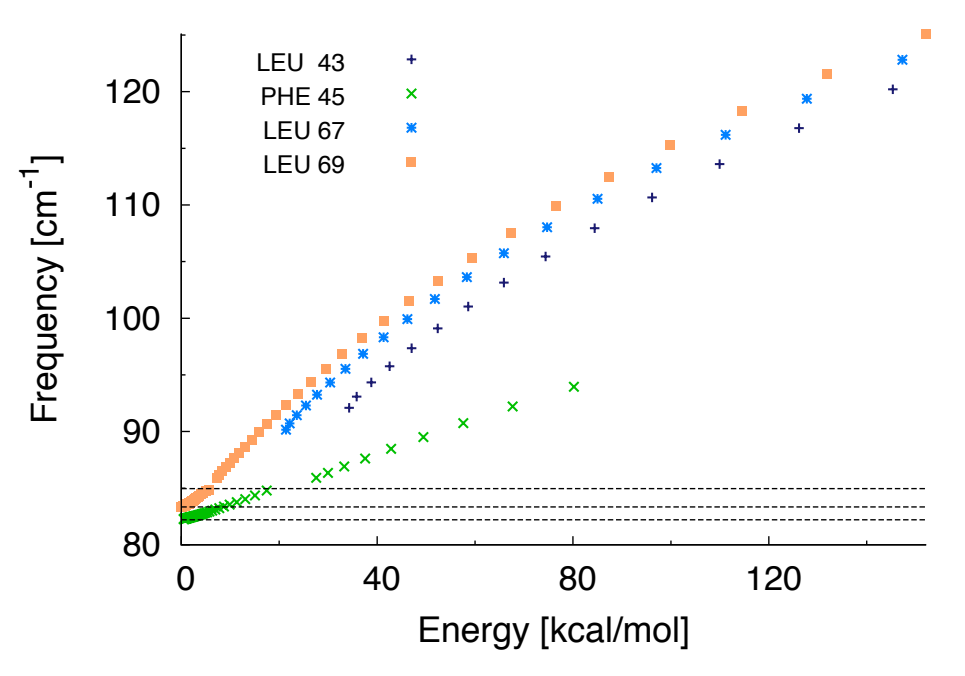

Figure 3. (Color online) Frequency vs energy for different Discrete Breathers computed in the 400-th conformer. The horizontal dashed lines mark the frequencies of the first edge normal modes.

conformers). It can be clearly appreciated that such DB modes could be regarded as plausible realizations of the experimentally detected motions, as the displacement patterns match to a remarkable extent the experimental observations.

As a general fact, the frequency and energy ranges where DBs can be found in a protein are broad and strongly site-dependent [64. The dispersion curves shown in Fig 3 make clear that pattern-matching DBs such as those illustrated in Fig. 2 can be excited at energies as low as $5-10 \mathrm{kcal} / \mathrm{mol}$ at selected sites with frequency above the linear spectrum. This places such modes among the most robust ones, as resonances with NMs are only possible with higher harmonics of linear modes. To be more precise, pattern-matching DBs such as those centered at PHE 45 and LEU 69 happen to fall within the special class of zero-gap modes, that is, they can be excited at arbitrary low energies, and can by all means be regarded as analytical continuations of band-edge normal modes that are stabilized through non-linear mechanisms T. Remarkably, this also means that they are among the most stable DBs - the more energy is injected the more localized and resilient they become 64. At low energies, such modes are in fact intra-band breathers, e.g. their frequencies fall within the gap between two successive normal modes. This is made possible by the discrete nature (finite number of particles) of the protein and constitutes one of the most distinctive features of localized nonlinear modes in discrete systems lacking translational symmetry 64.

As a general fact concerning breathers in protein structures [64, the majority of sites does not host zero-gap DBs, meaning that it can be exceedingly hard to excite a DB at a generic location, depending on the associated energy threshold. On the contrary, zero-gap DBs can only be found at very few special sites, usually lying within the stiffest regions of the structure 64]. It is thus tempting to speculate

I As a general feature, in the low-energy limit zero-gap DBs approach a given high-frequency bandedge normal mode. In the analyzed conformer, DBs at LEU 69 and PHE 45 approach the $2^{\text {nd }}$ and $3^{\text {rd }}$ NMs (from the band edge), respectively. 
that a particular biological relevance can be attached to the patterns of selected zero-gap DBs, which are likely to be excited spontaneously at $300 \mathrm{~K} 円$. Following Ref. [52, the average waiting time between spontaneously occurring uncorrelated energy fluctuations of magnitude $\epsilon$ can be estimated as

$$
\bar{\tau}_{\mathrm{th}} \simeq \frac{1}{2 \gamma} \sqrt{\frac{\pi}{\beta \epsilon}} e^{\beta \epsilon}
$$

where $\beta=1 / k_{B} T$ and $\gamma \simeq 1 \mathrm{ps}^{-1}$ is a typical damping coefficient specifying the decorrelation time scale of atomic tumbling. For fluctuations in the range $4 \div 5$ $\mathrm{kcal} / \mathrm{mol}$ at $T=300 \mathrm{~K}$, one gets $\bar{\tau}_{\text {th }} \simeq 0.1 \div 1 \mathrm{nsec}$. This means that it should be possible to observe the signature of a DB excited out of thermal fluctuations (at least) at sites LEU 69 and PHE 45. Therefore, the possibility that DB excitation may explain the correlated motions observed experimentally in Ref. [14 appears physically realistic.

\section{Discrete Breathers in the NMR ensemble}

We have seen that for a randomly selected conformer the vibrational patterns of DB modes centered at specific sites match to a surprising extent the correlated motion found experimentally. However, our calculations take a single conformer as the equilibrium structure for constructing the NNM. Therefore, it is necessary to investigate whether this is an isolated property displayed by DB modes in a few special conformers, or rather it reflects a general property of DB solutions over the ensemble of (experimentally determined) allowed conformations. We note that part of the answer is already known, as the few key sites where the interesting DBs appear lie within stiff regions. Therefore, they should retain similar spatial arrangements of their neighborhoods over the ensemble (see again Fig. 1). This should guarantee that similar DBs should be found at the same hinge-like locations in all the conformers.

To answer the above question, we introduce the following energy-dependent indicator for a specific DB whose vibrational pattern is $\boldsymbol{\xi}_{i}, i=1,2, \ldots, N$

$$
f_{A}=\sum_{i \in A}\left|\boldsymbol{\xi}_{i}\right|^{2} / \sum_{i=1}^{N}\left|\boldsymbol{\xi}_{i}\right|^{2}
$$

where $A$ is the subset of residues that participate to the long-lived experimental motion specified in (5). By construction, the quantity $f_{A}$ measures to what extent the pattern of a given $\mathrm{DB}$ (with a given energy) in a given conformer matches the experimentally highlighted structural correlation. It should be noted that $f_{A}$ only measures the geographical overlap, ignoring by construction the specific directions of the vibrational patterns to be compared, as this information is unfortunately not available from the experiments.

Of course, it could be objected that there exists a given probability that whatever pattern match a given region comprising five non-overlapping segments of the protein scaffold. For this reason, we have also calculated a $p$-value associated with such null hypothesis, i.e. a measure of statistical significance associated with the measured $f_{A}$

+ Of course, this does not mean that the vibrational pattern of all zero-gap DBs that may exist in a given protein must have a special biological significance. 


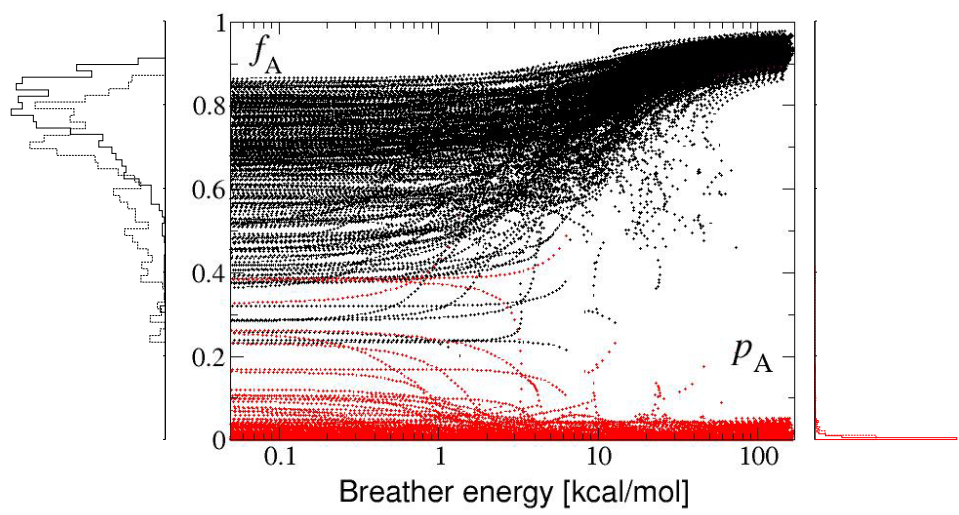

(a)

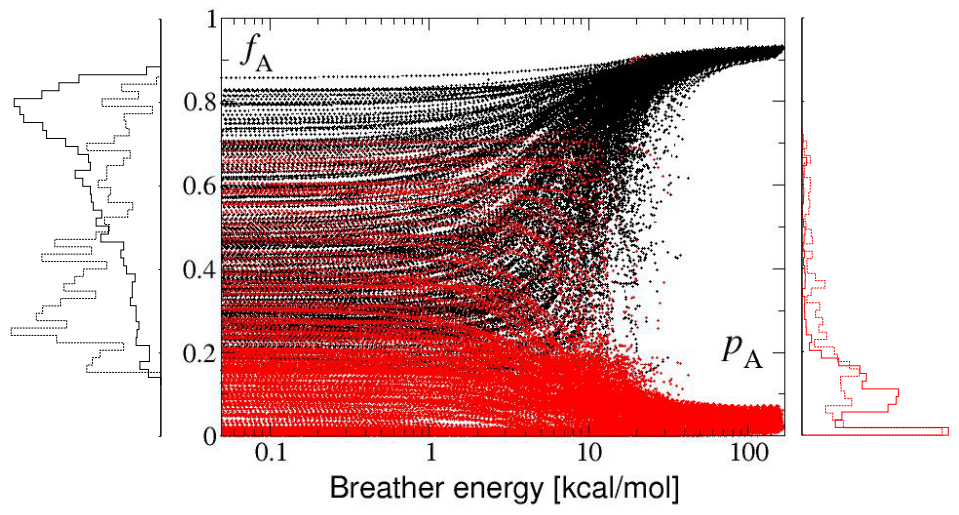

(b)

Figure 4. (Color online) Fraction of DB pattern covering the experimental correlated motion versus energy for a DB mode centered at LEU 69 (a) and LEU 50 (b). All DBs found in the entire database of 640 conformers are shown. The statistical significance of the vibrational pattern matching is illustrated through the probability $p_{A}$ (red points), Eq. (8) (see text). The side histograms show the distributions of $f_{A}$ and $p_{A}$ over the whole database for energies between 7 and $10 \mathrm{kcal} / \mathrm{mol}$ (thick solid lines) and for energies between 0.1 and $1 \mathrm{kcal} / \mathrm{mol}$ (thin dashed lines). 
values. To this aim, the following quantity has been also computed at all values of energies for all conformers

$$
p_{A}=\int_{f_{A}}^{1} \mathcal{P}(f) d f
$$

where the probability distribution $\mathcal{P}(f)$ has been reconstructed by generating a large number of random partitions, each consisting of a random permutation of five segments of fixed length (the same lengths as the segments in $A$ ) centered at as many random locations. The quantity $p_{A}$ is simply the probability that a given match score larger than the one actually observed would be observed for a random partition with the same structure as $A$. Thus, $p_{A}$ provides an estimate of the rejection probability associated with the corresponding $f_{A}$ value. The results of these analyses are shown in Figs. 4.

As a first global observation, it can be appreciated that the fraction of sizable pattern-matching scores is large over the whole ensemble of conformers. Furthermore, while at low energies DBs in different conformers display substantially variable scores, above $10 \mathrm{kcal} / \mathrm{mol}$ all DBs display the same large value of $f_{A}$. As the patternmatching score of DBs increases, it can be seen that this is so with increasing statistical confidence, as the corresponding rejection probability drops to zero. The case of the DB centered at LEU 69 provides a rather clear demonstration of this effect.

It is interesting to remark that in some cases the fingerprint of the experimental correlated pattern seems to be present in nuce already at the harmonic level. This can be clearly appreciated by comparing the histograms of $f_{A}$ values for DBs with energies between 0.1 and $1 \mathrm{kcal} / \mathrm{mol}$ and between 7 and $10 \mathrm{kcal} / \mathrm{mol}$. In the case of DBs localized at LEU 69, for example (Fig. 4 (a)), nonlinearity manifestly causes a pre-existing, low-energy signature to become sharper. As the DB becomes more energetic, its pattern captures to an increasing extent the experimental correlated motion. In this case, one may recognize nonlinear focussing of a pre-existent, foldencoded vibrational pattern.

However, as it is seen from Fig. 4 (b), nonlinearity is also able to promote a localized mode capturing the experimental pattern at intermediate and high energies without it showing significant traces in the harmonic regime. At variance with DBs localized at LEU 69, DBs centered at LEU 50 change substantially from low to intermediate energies, increasingly focussing their vibrational pattern within the experimental region $A$. In this case, a dynamical rearrangement occurs through sheer nonlinear effects, as a DB changes markedly its pattern to match the experimental vibration to an increasing extent when its energy builds up. This result demonstrates in a clear fashion that our nonlinear analysis is able to dig up information unavailable at the NM level.

Altogether, the physical properties of the same DBs in different conformers show to be highly consistent. This is demonstrated by the energy vs amplitude relations displayed in Fig. 5, which makes clear that the mean curves calculated by averaging the corresponding single-conformer relations over the ensemble are in excellent agreement with the properties of the average $D B$, i.e. the $\mathrm{DB}$ computed in the ensemble-averaged structure. Furthermore, we observe that the same gap-less DBs are present in a sizable fraction of the conformers (as e.g. in the case of the DB at LEU 69 whose pattern is shown in Fig. 22). Considering for example the DBs at LEU 69 and LEU 50, we find that $39 \%$ and $46 \%$ of the DBs over the ensemble, respectively, display a vanishing gap. The average excitation threshold for the rest of the breathers is (LEU 69) $\Delta E=25.8 \pm 0.6$ $\mathrm{kcal} / \mathrm{mol}$ and (LEU 50) $\Delta E=14.5 \pm 0.3 \mathrm{kcal} / \mathrm{mol}$ (see also insets in Fig. 5). All in 
all, it seems that it is easier to excite DBs whose pattern bears less resemblance to those of high-frequency normal modes.

\section{Conclusions}

In this paper we show that the puzzling features of a long-lived, highly correlated motion found in Ubiquitin through NMR measurements [14 match to a surprising extent the vibrational pattern of discrete breather modes centered at specific hot-spot sites. Our calculations show that such nonlinear modes could be excited spontaneously out of thermal fluctuations at room temperature. While this could be the first demonstration of the experimental detection of DB-like vibrations in a protein, it is at the same time a powerful demonstration of the ability of our nonlinear analysis [55] to predict relevant structure-spanning dynamical structures that may be central to the transmission of allosteric signals across protein scaffolds [12].

The emerging picture is that persistent concentration of energy along specific patterns could play a pivotal role in directing conformational changes at a higher level. One may speculate that protein folds might encode for the repeated excitation of long-lived bursts of higher-than thermal energy localized at specific hot spots located within stiff regions. Therefore, pattern-matching large-scale correlations embodied by collective modes could be effectively sustained by such long-lived nonlinear vibrations, that tend to self-focus where the nodes of the extended modes are. We note that this picture is intriguingly remindful of the interplay between nonlinear localized and delocalized motions found by Garcia in atomistic molecular dynamics trajectories of a small protein in the early 1990s [27.

In this scenario, already evoked in the context of enzyme functioning 29] and allosteric behavior [28, the node/hot spot pattern complementarity of specific slow and fast modes would lie at the core of protein functional dynamics. Specific, fold-encoded large-scale motions could be stabilized through nonlinear effects, thus causing the protein to privilege specific functionally relevant fluctuations, such as those of a binding pocket, hinged at some hot-spot site(s), that intermittently but steadily breaths open or analogous large-scale motions involving cross-talk between hinged domains. This picture is ultimately supported by general properties of discrete breathers: DBs are not only known for their resilience to perturbations, lowdamping rate due to reduced contact with the solvent and hindered resonant energy transfer with NMs. Remarkably they display the unique property of self-sustaining by harvesting energy from the background [63, which has been recently demonstrated to also occur in proteins [51.

In summary, our study prompts the intriguing hypothesis that unusually long-lived DB-like modes might be central to rationalize how protein folds encode intramolecular cross-talk. As such, DB-based analyses could provide a key computational method to identify unknown dynamical structures at the core of allosteric transduction mechanisms in proteins.

\section{Acknowledgements}

The author is deeply thankful to R. Nussinov, P. Csermely, Y.-H. Sanejouand and P. De Los Rios for a critical reading of this manuscript and for their most enlightening comments. The author is also indebted to R. B. Fenwick, L. Orellana and X. Salvatella for illuminating discussions concerning the application of the DB-based method to 


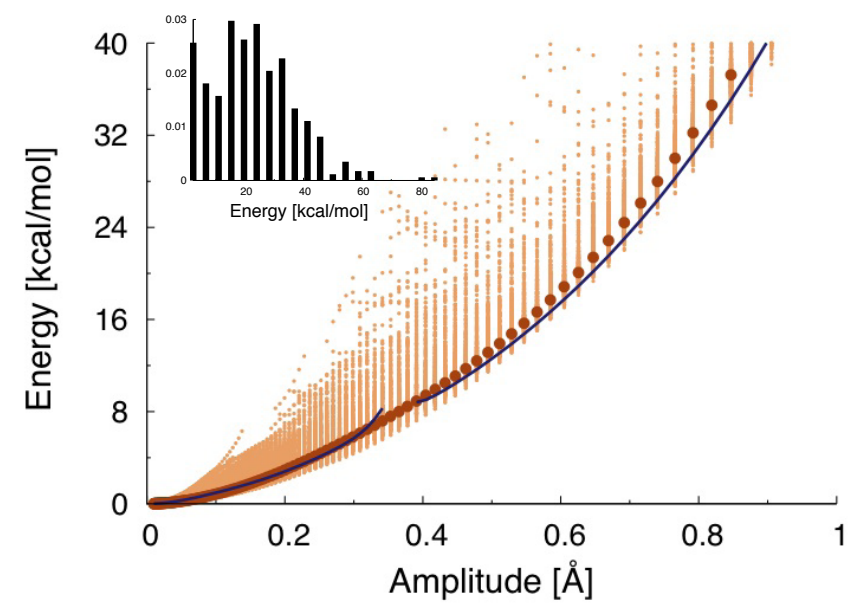

(a)

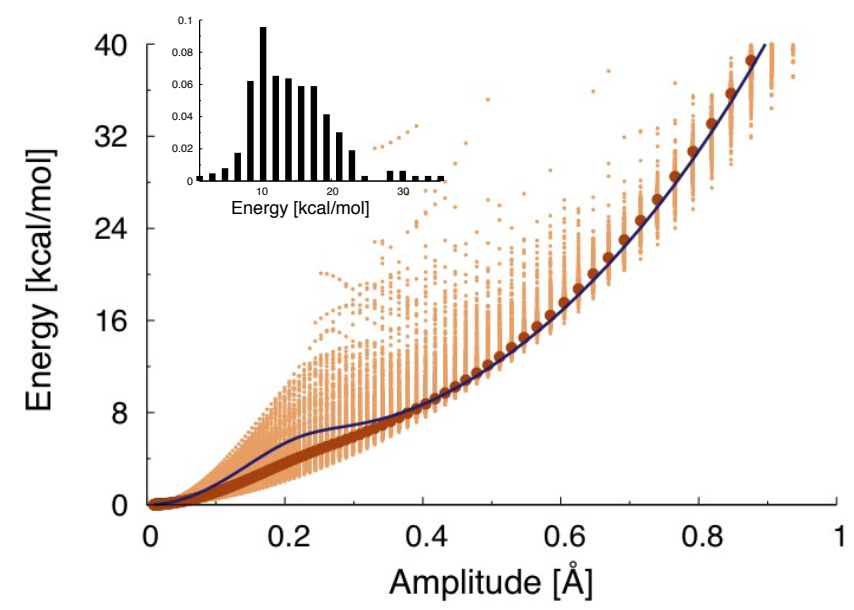

(b)

Figure 5. (Color online) Amplitude versus energy for Discrete Breathers computed in all 640 conformers and centered at (a) LEU 69 and (b) LEU 50 (light filled circles). The dark filled circles represent the corresponding average curve. The solid line refers to the DB computed in the average structure computed over the NMR ensemble of conformers. The insets show the histograms of the energy gaps calculated over the whole ensemble.

their NMR data. Finally, the author would like to thank L. Turin for making him aware of the inspiring ideas developed in the early 1970s by C. W. F. McClare. The author acknowledges financial support from the EU-FP7 project PAPETS (GA 323901).

\section{References}

[1] Changeux J P and Edelstein S J 2005 Science 308 1424-1428 
[2] Kern D and Zuiderweg E R 2003 Current Opinion in Structural Biology 13 748757

[3] Gunasekaran K, Ma B and Nussinov R 2004 Proteins: Structure, Function, and Bioinformatics 57 433-443

[4] Bahar I and Jernigan R L 1999 Biochemistry 38 3478-3490

[5] Marques O and Sanejouand Y H 1995 Proteins 23 557-560

[6] Li G and Cui Q 2004 Biophys. J. 86 743-763

[7] Parak F G 2003 Reports on Progress in Physics 66103

[8] Rambo R P and Tainer J A 2013 Annual Review of Biophysics 42 415-441

[9] Zaccai G 2012 European Biophysics Journal 41 781-787

[10] Bu Z and Callaway D J 2011 Protein Structure and Diseases (Advances in Protein Chemistry and Structural Biology vol 83) ed Donev R (Academic Press) pp 163 $-221$

[11] Akimoto M, Selvaratnam R, McNicholl E T, Verma G, Taylor S S and Melacini G 2013 Proceedings of the National Academy of Sciences 110 14231-14236

[12] Selvaratnam R, Chowdhury S, VanSchouwen B and Melacini G 2011 Proceedings of the National Academy of Sciences 108 6133-6138

[13] Kalodimos C G 2011 Protein Science 20 773-782

[14] Fenwick R B, Esteban-Martín S, Richter B, Lee D, Walter K F A, Milovanovic D, Becker S, Lakomek N A, Griesinger C and Salvatella X 2011 Journal of the American Chemical Society 133 10336-10339

[15] Ishima R and Torchia D A 2000 Nature Structure Molecular Biology 7 740-743

[16] Das R, Chowdhury S, Mazhab-Jafari M T, SilDas S, Selvaratnam R and Melacini G 2009 Journal of Biological Chemistry 284 23682-23696

[17] Das R, Mazhab-Jafari M T, Chowdhury S, SilDas S, Selvaratnam R and Melacini G 2008 Journal of Biological Chemistry 283 19691-19703

[18] Csermely P, Palotai R and Nussinov R 2010 Trends in Biochemical Sciences 35 $539-546$

[19] Leitner D M 2008 Annual Review of Physical Chemistry 59 233-259

[20] Moritsugu K, Miyashita O and Kidera A 2000 Phys. Rev. letters 85 3970-3973

[21] Tama F and Sanejouand Y H 2001 Prot. Eng. 14 1-6

[22] Kim M K, Jernigan R L and Chirikjian G S 2002 Biophysical journal 83 16201630

[23] Ma J and Karplus M 1997 J. Mol. Biol. 274 114-131

[24] Zheng W and Brooks B R 2005 Biophys. J. 88 3109-3117

[25] McCammon J A and Harvey C S 1987 Dynamics of proteins and nucleic acids (New-York: Cambridge University Press)

[26] Meinhold L, Smith J C, Kitao A and Zewail A H 2007 Proceedings of the National Academy of Sciences 104 17261-17265

[27] García A E 1992 Physical Review Letters 68 2696-2699

[28] Hawkins R J and McLeish T C B 2006 Biophys. J. 91 2055-2062

[29] Yang L W and Bahar I 2005 Structure 13 893-904 
[30] Cooper A and Dryden D T F 1984 European Biophysics Journal 11 103-109

[31] Bahar I, Atilgan A R, Demirel M C and Erman B 1998 Physical Review Letters $802733-2736$

[32] McClare C W F 1972 Journal of Theoretical Biology 35 233-246

[33] McClare C W F 1972 Journal of Theoretical Biology 35 569-595

[34] McClare C W F 1975 Energy Transformation in Biological Systems Ciba Foundation Symposium 31 (New Series) (Amsterdam, Oxford, New York: Elsevier - North-Holland) pp 301-325

[35] Whitford P C, Onuchic J N and Wolynes P G 2008 HFSP Journal 2 61-64

[36] Eisenmesser E Z, Bosco D A, Akke M and Kern D 2002 Science 295 1520-1523

[37] Henzler-Wildman K A, Lei M, Thai V, Kerns S J, Karplus M and Kern D 2007 Nature 450 913-916

[38] Yu X and Leitner D 2003 Journal of Physical Chemistry B 107 1698-1707

[39] Woutersen S and Hamm P 2002 Journal of Physics: Condensed Matter 14 R1035R1062

[40] Xie A, van der Meer L, Hoff W and Austin R H 2000 Physical Review Letters 84 $5435-5438$

[41] Xie A, van der Meer A F G and Austin R H 2001 Physical Review Letters 88 018102

[42] d'Ovidio F, Bohr H G and Lindgrd P A 2005 Physical Review E 71 026606-9

[43] Scott A 1992 Physics Reports 217 1-67

[44] Archilla J F R, Gaididei Y B, Christiansen P L and Cuevas J 2002 Journal of Physics A 35 8885-8902

[45] Kopidakis G, Aubry S and Tsironis G P 2001 Phys. Rev. Lett. 87

[46] Levy R, Perahia D and Karplus M 1982 Proc. Natl. Acad. Sci. USA 79 1346-1350

[47] Hayward S, Kitao A and Go N 1995 Proteins 23 177-186

[48] Peyrard M (ed) 1995 Nonlinear excitations in biomolecules (Springer: Berlin)

[49] Sagnella D, Straub J and Thirumalai D 2000 J. Chem. Phys. 113 7702-7711

[50] Roh J H, Novikov V N, Gregory R B, Curtis J E, Chowdhuri Z and Sokolov A P 2005 Physical Review Letters $95038101-$

[51] Piazza F and Sanejouand Y H 2009 Europhysics Letters 8868001

[52] Piazza F and Sanejouand Y H 2011 Discrete and Continuous Dynamical Systems, Series $S 41247$ - 1266

[53] Flach S and Gorbach A V 2008 Physics Reports 467 1-116

[54] Luccioli S, Imparato A, Lepri S, Piazza F and Torcini A 2011 Physical Biology 8 046008

[55] Juanico B, Sanejouand Y H, Piazza F and De Los Rios P 2007 Phys. Rev. Lett. 99238104

[56] Bruschweiler R, Liao X and Wright P 1995 Science 268 886-889

[57] Bax A and Grzesiek S 1993 Accounts of Chemical Research 26 131-138

[58] Zhuravleva A and Gierasch L M 2011 Proceedings of the National Academy of Sciences 108 6987-6992 
[59] Vendruscolo M 2011 Nat Chem Biol 7 411-412

[60] Sukhorukov A A, Kivshar Y S, Bang O, Rasmussen J J and Christiansen P L 2001 Phys. Rev. E 6303036601

[61] Rasmussen K O, Cai D, Bishop A R and Gronbech-Jensen N 1999 Europhysics Letters 47 421-427 ISSN 0295-5075

[62] Kopidakis G and Aubry S 2000 Physica D: Nonlinear Phenomena 139 247-275

[63] Flach S and Willis C R 1998 Phys. Rep. 295 181-264

[64] Piazza F and Sanejouand Y H 2008 Physical Biology 5026001 